\title{
Italique
}

Poésie italienne de la Renaissance

II | 1999

Varia

\section{Armida e Proteo. Un percorso tra Gerusalemme Liberata e Conquistata}

Matteo Residori

\section{(2) OpenEdition}

Edizione digitale

URL: https://journals.openedition.org/italique/202

DOI: 10.4000/italique.202

ISSN: 1663-4438

\section{Editore}

Librairie Droz

\section{Edizione cartacea}

Data di pubblicazione: 1 décembre 1999

Paginazione: 113-142

ISBN: 2-600-00389-4

ISSN: 1423-3983

Notizia bibliografica digitale

Matteo Residori, «Armida e Proteo. Un percorso tra Gerusalemme Liberata e Conquistata», Italique

[Online], II | 1999, online dal 07 octobre 2009, consultato il 24 novembre 2022. URL: http://

journals.openedition.org/italique/202 ; DOI: https://doi.org/10.4000/italique.202

All rights reserved 
MATTEO RESIDOR I

A RMIDA E PROTEO.

U N PERCORSO TRA GERUSALEMME LIBERATA E CONQUISTATA 


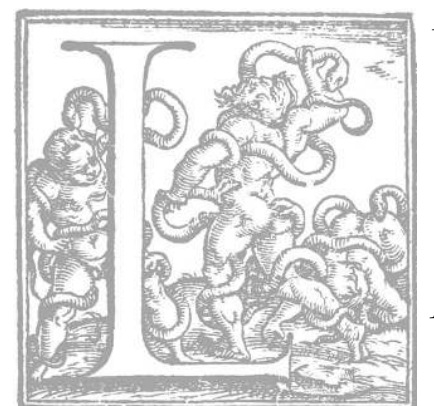

A descrizione delle arti seduttive di Armida nel IV e $V$ canto della Gerusalemme Liberata, facendo del suo personaggio l'incarnazione di un artificio retorico che coniuga molteplicità e incostanza, le assegna una posizione nevralgica nel sistema simbolico del poema tassiano. Nelle pagine che seguono si cercherà di precisare la natura di questo artificio, e di seguire le costanti simboliche associate ad Armida lungo un percorso che attraversa alcune opere della maturità tassiana per approdare infine alla Conquistata. Si tratterà in particolare di mostrare attraverso quali riferimenti culturali l'autore articoli il nucleo tematico della metamorfosi, che è molto prossimo a quell'opposiżione tra uniforme e multiforme che innerva - come ha mostrato l'importante studio di Sergio Zatti - l'intero tessuto della Gerusalemme. ${ }^{\mathrm{I}}$ Come si vedrà, la persistente centralità di questi temi nell' ultima fase dell'opera tassiana incoraggia a leggere il destino narrativo di Armida nella Conquistata come un'espressione esemplare delle preoccupazioni e degli scrupoli che guidano la 'riforma' del poema.

Il successo di Armida nel campo crociato dipende dall" " arte 》 e dall" "ingegno» anche più che dalla "beltà». "Quo non ars penetrat? " si chiedeva il poeta dell'Ars amatoria (III 29I); e la maga mostra la stessa fiducia nell'onnipotenza dell'ars, della sedurione intesa come tecnica e strategia retorica, mettendo abilmente in pratica molti dei consigli che Ovidio impartiva agli aspiranti seduttori nel suo poemetto didascalico. Il silenzio può essere più eloquente delle parole; le lacrime, suscitate e frenate a tempo opportuno, sono un'arma formidabile; occultare le bellezze è più efficace che mostrarle; la gelosia mantiene vivace l'amore. ${ }^{2}$ Ma ciò che più importa, ai fini del nostro discorso, è che l'intero episodio appaia dominato, fin nella sua struttura, da uno dei precetti più generali del libro ovidiano, quello che raccomanda al seduttore una versatilità all'altezza della varietà delle sue prede :

finiturus eram, sed sunt diversa puellis pectora; mille animos excipe mille modis. nec tellus eadem parit omnia : uitibus illa convenit, haec oleis, hic bene farra virent. pectoribus mores tot sunt, quot in ore figurae : qui sapit, innumeris moribus aptus erit, utque leues Proteus modo se tenuabit in undas, nunc leo, nunc arbor, nunc erit hirtus aper.

hi iaculo pisces, illi capiuntur ab hamis, 
hos caua contento retia fune trahunt.

Nec tibi conuenient cunctos modus unus ad annos;

longius insidias cerua videbit anus.

Si doctus videare rudi petulansue pudenti, diffidet miserae protinus illa sibi.

inde fit ut, quae se timuit committere honesto, uilis ad amplexus inferioris eat.

(Ars amatoria I 755-70)

Puntando a conquistare "mill'alme e mille 》 (IV 96), anche Armida calibra la sua strategia sullindole dei singoli crociati : "né con tutti, né sempre un stesso volto / serba, ma cangia a tempo atti e sembiante » (IV 87). Alla fine del IV canto il racconto si fa all'improvviso temporalmente vago e 'plurale'; gli eventi non sono riferiti nella loro successione cronologica, ma si dispongono secondo una griglia che corrisponde alla molteplicità dei tipi umani e alla varietà delle circostanze : "or ... or 》, "in quegli ... in questi ... », "Se scorge alcun ... Ad altri poi ... »; sembra di assistere all' azione simultanea di tante Armide diverse : benigna e incoraggiante con i timidi, ritrosa e severa con gli audaci, "rozza e inesperta 》 con i corteggiatori troppo intraprendenti. Come un retore consumato, la maga sa che il discorso persuasivo non ha come criterio la verità, ma si modella, per affinità o per contrasto, sulle qualità del destinatario; e, coerentemente, assume tanti volti quante sono le vittime designate. L'effetto, per il lettore, è quello di una "pura successione fenomenica di 'apparenze'», 3 e quasi di un'illusione ottica, di una visione a prospettiva multipla : l'immagine della donna cambia a seconda del punto di vista di chi la osserva, e tra tante immagini diverse è impossibile individuarne una che sia più vera delle altre. Per di più, il suo virtuosismo metamorfico non si esaurisce nel declinare sapientemente una casistica etica: ad ognuno dei crociati, infatti, la maga offre uno spettacolo di incostanza e sfrenata variabilità, passando senza sosta dallo sdegno alla pietà, dalla confidenza al distacco, dal pianto al riso. La somma di queste due forme di 'molteplicità' è l'incertezza assoluta, la vertigine conoscitiva: "Fra si contrarie tempre, in ghiaccio e in foco, / in riso $e$ in pianto, e fra paura e spene, / inforsa ogni suo stato » (IV 93); dove il confronto con il modello (Rvf. I52, 3-4 «in riso e 'n pianto, fra paura et spene / mi rota si ch'ogni mio stato inforsa ") mostra chiaramente che, trasferendo la metamorfosi dall'amante alla donna, Tasso tende a oggettivare eticamente il motivo dell'incostanza amorosa, a esaltare le componenti di artificio e di attiva illusione in una fenomenologia ossimorica peraltro ben petrarchesca. ${ }^{4}$ Tante metamorfosi non tardano a evocare il paragone con Proteo che si trovava già in Ovidio; siamo nel $V$ canto, nel momento in cui Armida cerca di sedurre addirittura Goffredo: 
Armida e Proteo

Tentò ella mill'arti, e in mille forme quasi Proteo novel gli apparse inanti, e desto Amor, dove più freddo ei dorme, avrian gli atti dolcissimi e i sembianti

(GL V 63)

Il tentativo, naturalmente, fallisce ("grazie divine »!). Ma non per questo Armida perde fiducia nelle virtù della metamorfosi: sul mar Morto (X 66-68), convertendo $i$ suoi prigionieri in pesci e minacciandoli di altre temibili trasformazioni, dà prova di conoscere anche le arti di Circe, figura perfettamente complementare a quella di Proteo. ${ }^{5}$ E non si può dimenticare la terribile metamorfosi nella selva degli incanti, dove non sarà un caso che Armida prenda le sembianze di Briareo, il mostro 'plurimo' per eccellenza (XVIII 35-36) : le « cinquanta spade » $i$ « cinquanta scudi 》 con cui affronta Rinaldo concretizzano iperbolicamente l'attacco molteplice che la maga aveva sferrato con le sue armi retoriche all'esercito cristiano. ${ }^{6}$ Ma è forse più importante che questa natura proteiforme segua Armida anche al di fuori degli spazi della magia e dell'offensiva strategica; e che anzi sembri il motore della formidabile vitalità narrativa che il personaggio dispiega, esaurito il suo compito epico, lungo il percorso marginale e privato che esso traccia nella seconda metà del poema, modellandolo a distanza su quello di Rinaldo, ormai unico, insensibile destinatario delle sue arti : è grazie alle sue risorse metamorfiche che la donna può reagire con rabbiosa energia all' abbandono dell'amato e tornare - ma ora come eroina elegiaca, estranea alla dimensione collettiva del conflitto sul campo di battaglia ("Ritentar ciascun'arte e trasmutarmi / in ogni forma insolita mi giova, / trattar l'arco e la spada, e serva farmi / de' più potenti e concitargli a prova », XVI 73); ${ }^{7}$ ed è a esse che tenta di ricorrere anche alla fine della battaglia campale, subito dopo aver constatato l'inutilità di tutte le sue precedenti trasformazioni (" e inerme io vinta sono, e vinta armata:/ nemica, amante, egualmente sprezzata.// Or qual arte novella e qual m'avanza / nova forma in cui possa anco mutarmi?", XX 66-67). Del resto, non è a un estremo sussulto del Proteo che si nasconde in Armida che dobbiamo anche la sua sconcertante 'conversione' finale (XX I36), l'improvvisa e incongrua metamorfosi in una sottomessa vergine Maria?

La volubilità metamorfica, esercitata su se stessa e sugli altri, è sicuramente il tratto del personaggio di Armida a cui più si deve la sua prodigiosa fortuna nella letteratura seicentesca, quella italiana e francese in particolare. E non si può negare che uno degli aspetti fondamentali della sensibilità barocca, come la descrive il classico libro di Jean Rousset, sia annunciato dalla viva attenzione di Tasso per la molteplicità, l'incostanza e la mutevolezza illusionistica del 
mondo naturale e umano, insomma dalla sua "gnoseologia ... sempre pericolosamente in bilico tra vero e falso, tra finzione e realtà $\gg .{ }^{8}$ D'altra parte, più di uno studio importante - ricordo in particolare quelli di Giamatti, Quint e Jeanneret - ha mostrato quale rilievo abbia già nell' immaginario rinascimentale il mito di Proteo, per il quale Tasso mostra, come vedremo, un interesse non occasionale. ${ }^{9}$ Chi segua anche sommariamente lo sviluppo della carriera letteraria tassiana dopo la Liberata incontrerà ancora numerose attestazioni di questo interesse. Ma potrà anche osservare le tracce di una significativa scomposizione del motivo : da un lato la metamorfosi è rappresentata con neutralità etica, se non con euforia, come principio a cui si devono la bellezza e la meraviglia del mondo; dall'altro essa è sottoposta a un'interpretazione morale, che ne evidenzia gli aspetti caotici e angosciosi e che porta a identificarla con la menzogna sofistica, nemica capitale della verità e della convivenza civile. Non si può negare che la descrizione delle arti di Armida rimanga, nonostante l'insistenza sul loro carattere ingannevole, piuttosto ambigua: la strategia retorica vi sconfina nellillusionismo magico, e ha insieme la leggerezza di una danza vorticosa, da cui il narratore è il primo a mostrarsi disorientato e sedotto; in questo Proteo non sono distinguibili il sofista, il mago e il ballerino, e le sue trasformazioni non fanno che esaltare il carattere di mobile illusione che è intrinseco alla natura stessa dell' amore e del mondo. ${ }^{10}$ Il Tasso delle opere più tarde pretenderà invece di distinguere, di tenere ben separate verità e menzogna, illusione mutevole e stabile realtà. Che conseguenze questo abbia, in particolare sul destino di Armida nel rifacimento della Gerusalemme, si cercherà di mostrarlo attraverso un percorso che prenderà in esame le apparizioni di Proteo e di alcune sue controfigure nell'opera tassiana.

\section{Proteo a teatro}

Il testo dell'Aminta è accompagnato, a partire da un'edizione postuma del I666, da un gruppo di intermedi di argomento mitologico. Per quanto essi non siano attestati da autografi, si tende a dare fiducia all'attribuzione seicentesca, e a ritenere che Tasso li abbia composti per una rappresentazione

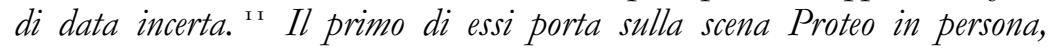
attribuendogli un ruolo che potremmo chiamare di supervisore alla scenografia:

Proteo son io, che trasmutar sembianti e forme soglio varïar sì spesso ; e trovai l'arte onde notturna scena 
Armida e Proteo

\author{
cangia l'aspetto ; e quinci Amore istesso \\ trasforma in tante guise i vaghi amanti, \\ com' ogni carme ed ogni storia è piena. \\ Ne la notte serena,

Il dio marino istituisce un significativo parallelo tra le trasformazioni illusorie che la sua arte opera a teatro e quelle che, mettendosi al servizio di Amore, essa produce sugli innamorati : il che andrà inteso come allusione alla peripezia drammatica dell'Aminta (in particolare alla conversione di Silvia), ma soprattutto alle metamorfosi in cui consiste topicamente la patologia amorosa; le stesse, insomma, che Armida-Proteo, "inforsando se stessa ", infliggeva ai suoi ammiratori nella Liberata. D'altra parte, anche Armida era capace di superbe messe in scena, di preferenza notturne: da quella nel castello sul mar Morto ("Splende il castel come in teatro adorno / suol fra notturne pompe altera scena, / ed in eccelsa parte Armida siede, / onde senz'esser vista e ode e vede ", VII 36) a quelle con cui cerca, con o senza successo, di irretire Rinaldo ("Cosi dal palco di notturna scena / o ninfa o dea, tarda sorgendo, appare », XIV 6I ; "Quai le mostra la scena o quai dipinte / tal volta rimiriam dèe boscareccie, / (...) / tal in sembianza si vedean le finte / figlie de le salvatiche corteccie 》, XVIII 27) ; per non dire del palazzo e del giardino alle Isole Fortunate, che hanno nel complesso il carattere vistoso ed effimero di una gigantesca scenografia teatrale. Nello spazio istituzionalmente metatestuale dell' intermedio, insomma, Armida vede riconosciuta indirettamente l'importanza del suo ruolo di regista di un'azione che - parallela e ostile a quella epica - deriva non poca della sua efficacia ipnotica dalla rapidità con cui cambiano le maschere e si alternano gli scenari.

\title{
Le metamorfosi del bello
}

Il paragone che qualificava le arti di Armida nel $V$ canto della Liberata ("Quasi Proteo novel») suggerisce l'attacco a due madrigali gemelli, collocati nelle edizioni moderne tra le rime amorose estravaganti : 
Come sia Proteo o mago,

il bello si trasforma e cangia imago :

or si fa bianco or nero

in due begli occhi, or mansueto or

or in vaghi zaffiri

fa con Amor soavi e lieti giri;

or s'imperla or s'inostra,

or ne le rose ed or ne le viole

d'un bel viso ei si mostra;

ora stella somiglia, or luna, or sole ;

talor per gran ventura

egli par il Silenzio a notte oscura.

(Rime 268)
Quasi Proteo novello,

in varie forme si trasmuta il bello:

or sembra luna, or sole,

or la vermiglia aurora,

or ninfa in mare, o qui Pomona o

Flora ;

or ne le rose ed or ne le viole,

or avvien che si miri

nel color de' giacinti o de' zaffiri;

or vento pare, or fiamma,

or neve e gelo; e pur co 'l gelo in-

fiamma.

Nel primo madrigale, la griglia temporale plurima che organizzava la descrizione delle arti di Armida (" or ... or») diventa la struttura portante del testo: ma essa, più che esprimere la mobilità psicologica dell'amata (salvo che in "or mansueto or fero", che riprende Rvf. II2, 8 "or mansüeta, or disdegnosa et fera»), serve a dare una singolare rappresentazione dinamica delle sue attrattive fisiche : gli elementi topici della descriptio lirica non compongono un quadro unitario, ma sono dati come alternativi e successivi, come altrettante incarnazioni del «bello» trasformista. Persino il contrasto tra il «bianco » e il «nero» degli occhi è il risultato di una performance virtuosistica di questo Proteo-camaleonte; e una polarità simile presiede alla enigmatica trasformaz̧ione finale : da sole a Silenz̧io notturno, la divinità favorevole agli amanti. La bellezza, insomma, è intrinsecamente mobile, multiforme, molteplice. E se già in questo madrigale un metaforismo piuttosto fluido e vago tende a identificare questa multiformità con quella che caratterizza l'intera natura, nel secondo la scomparsa di ogni accenno all' illustrandum umano e una maggiore ricettività verso $i$ cataloghi classici delle trasformazioni di Proteo (cfr. per esempio Georg. IV 409-10 « aut acrem flammae sonitum dabit atque ita vinclis / excidet") ${ }^{14}$ mettono in primo piano la varietà dispiegata del mondo naturale; almeno fino a quando la pointe finale non ci riporta all' ambito dei paradossi amorosi.

Il secondo madrigale fa parte di un gruppo di trentasei che Tasso compose alla fine del I592 per Carlo Gesualdo, principe di Venosa. È degno di nota che l'immagine di Proteo appaia in filigrana anche nella prima lettera che accompagna l'invio dei testi al musicista : l'infermità e la persecuzione della fortuna hanno molto impoverito l'ingegno dell'autore, il quale si è sforzato tuttavia "di trasmutarsi in nuove forme, com'è conveniente al poeta : il quale, per 
opinione d'Aristotile, o deve essere divino, o di pieghevole ingegno " ; ${ }^{\text {is }}$ con allusione a un celebre e controverso passo della Poetica in cui Aristotele raccomanda all'autore scenico di trasformarsi via via in ciascuno dei suoi personaggi, per poter dare una rappresentazione efficace delle loro passioni. ${ }^{16}$ D'altra parte, in una lettera di qualche tempo dopo allo stesso Gesualdo la versatilità del poeta amoroso si precisa come una facilità di invenzione degna del suo tema: "L'esperienza m'ha fatto vergognare di me stesso, e del mio ingegno poco pronto al ritrovare, ed assai povero ne la copia di cose infinite che si posson dire de la bellezza ». ${ }^{17}$ Le metamorfosi del bello e quelle della poesia sono dunque legate da un rapporto di stretta implicazione: la mutevolezza del bello dipende da quella del poeta, il quale realiza appieno il suo compito quando esalta la cangiante multiformità delle apparenze.

Un'ampia trattazione di questo tema, dai due punti di vista complementari, si trova nei due dialoghi Il Minturno (I593) e Il Malpiglio secondo (I585), dove viene anche esplicitato lo sfondo filosofico, essenzialmente platonico, della riflessione tassiana. In entrambi $i$ casi il punto di partenza è la lirica del Petrarca, letta come espressione esemplare di 'molteplicità' poetica. Nel Minturno, dedicato interamente al tema della bellezza, si osserva come il poeta lirico trovi ogni volta ragioni diverse per lodare la sua donna, e non arretri neppure di fronte alle contraddizioni : ora ne loda $i$ capelli biondi, ora il pallore; ora la ama altera, ora gentile. Questa volubilità è la prima fonte del carattere multiforme del bello, la seconda essendo la molteplicità delle indoli e dei gusti umani (" altri s'invaghiscono de gli occhi azzurri; ad altri sogliono piacere $i$ negri maggiormente; a molti la severità diletta, a molti la mansuetudine 》, $\int$ 50), la stessa di cui Armida aveva saputo tenere conto cosi abilmente nella sua opera di seduzione plurima. A giusto titolo, allora, il bello può essere paragonato a un Proteo naturale, il camaleonte : " il bello sarà trasmutabile, e a guisa di camaleonte prenderà diversi colori, diverse forme e diverse imagini e apparenze 》 (』 SI). ${ }^{18}$ Questa concezione volubile e prospettica, basata sull'unico criterio del " gusto», è l'essenza stessa della lirica d'amore, il nucleo della sua "varietà » e delle sue potenzialità metaforiche : e non è necessario uscire dal gruppo di madrigali per Gesualdo da Venosa per veder dispiegate da Tasso tutte le risorse del genere ben oltre il canone petrarchesco, fino al paradosso squisitamente soggettivo delle lodi a una donna " non bella » che sa rendere bella ogni cosa sgradevole. ${ }^{19} \mathrm{Ma}$ il dialogo non tarda a introdurre la contrapposizione platonica tra il bello mutevole e contingente e il bello in sé, stabile e unitario; e se il discorso prosegue attraverso l'assimilazione canonica della molteplicità estetica al disordine morale e all'anarchia politica, non puì sfuggire che lungo tutto il testo circola un altro capitale motivo platonico : il sospetto verso la poesia, avvicinata all'attività sofistica di ingannevole moltiplicazione delle immagini. 
Da questo tema aveva preso l'avvio il Malpiglio secondo, che, come annuncia il sottotitolo (del fuggir la moltitudine), è strutturato come una ricerca del modo di sottrarsi all' angosciosa 'moltitudine' che assedia l'uomo di lettere fin nella solitudine del suo studio. L'esempio della poesia di Petrarca serve a introdurre un discorso sulle due forme di disturbante molteplicità che derivano dalla lettura dei poeti : quella delle passioni, prodotta dall'adesione emotiva che le opere promuovono in chi legge ("Co 'l Petrarca dunque vi rallegrate e dolete e temete ancora e sperate ... laonde oltre una multitudine di sensi interiori e d'imaginazioni avete, o più tosto abbiamo ne l'animo un gran numero di passioni $\gg, \int$ 9-Io), e quella delle opinioni, che - esattamente come la molteplicità della bellezza - deriva tanto dal gran numero di autori letti quanto dalla volubilità che è propria di ciascuno di essi ("s'in un autore medesimo e s'intorno ad uno soggetto istesso troviamo alcuna volta gran diversità di pareri, quanta maggiore si può ritrovare in tanti scrittori e si diversi ... », $\left.\int I 6\right) .{ }^{20}$ La letteratura nel suo complesso (il discorso riguarda a questo punto "poeti, oratori e istorici») alimenta dunque il mare tempestoso della 'moltitudine', e la sua frequentazione è fortemente sconsigliata a chi desidera rifugiarsi nel porto tranquillo della verità, che per sua essenza è una. La critica platonica sta naturalmente sullo sfondo di tutta la riflessione letteraria di Tasso; ma direi che è solo a partire dagli anni '8o, con $i$ Dialoghi, $i$ nuovi Discorsi del poema eroico e gli scritti intorno al poema, che essa viene assunta - anche attraverso la mediazione della patristica - in tutta la sua paralizzante radicalità. La Gerusalemme Conquistata, il 'poema riformato', è senz'altro l'opera in cui si esprime più coerentemente il tentativo di affidare alla poesia l'«antidoto» contro il "veleno » che essa stessa secerne: ${ }^{21}$ l'ultima tappa del nostro percorso sulle tracce di Proteo, ambiguo sofista ma anche nume tutelare della "varietà 》 poetica, ci fornirà un esempio eloquente del modo in cui questa impresa paradossale può riuscire.

\section{I colori della retorica}

Nel rifacimento della Gerusalemme, a cui Tasso lavora dal I588 al I592, la prima parte della vicenda di Armida (quella che arriva fino alla liberazione di Rinaldo, ora chiamato Riccardo) subisce pochi e isolati ritocchi: la rassegna delle sue arti rimane più o meno intatta; l'episodio del castello sul Mar Nero viene depurato dell'eccesso di 'meraviglioso' della trasformazione dei crociati in pesci; mentre nella lunga sezione degli amori con Rinaldo/Riccardo non sembra di scorgere mutamenti di rilievo, a parte lo spostamento della scena- 
Armida e Proteo

dettato dallo scrupolo dell'unità di luogo - dalle Isole Fortunate al monte Libano. Eppure, proprio in quest'ultimo episodio si concentrano alcuni interventi che hanno a che fare con la natura proteiforme di Armida, e che sembrano coerenti con quello che è indubbiamente l'elemento più vistoso del destino del personaggio nella Conquistata : la sua completa scomparsa dalla seconda metà del poema. ${ }^{22}$

Il trasferimento del regno della maga comporta naturalmente lo stralcio del viaggio oceanico dei due crociati Carlo e Ubaldo (ora Ruperto e Araldo), e la scomparsa del personaggio che li guidava alla meta sulla sua barchetta: una personificazione della Fortuna, la cui collaborazione al piano provvidenziale non può del resto che apparire impropria a un autore che, oltre a essere sempre più preoccupato della correttezza teologica delle sue invenzioni, ba eletto la dea incostante sua nemica personale. La donzella allegorica, però, lascia in eredità ad Armida il più vistoso dei suoi attributi, una gonna di colore cangiante:

La sua gonna or cerulea ed or vermiglia

diresti, ed or s'indora ed or verdeggia ;

sì ch'uom sempre diversa a sé la vede, quantunque volte a riguardarla riede.

Così piuma talor, che di gentile amorosa colomba il collo cinge, mai non si mostra a se stessa simile, ma 'n diversi colori al sol si tinge: or d'accesi rubin sembra un monile, or di verdi smeraldi il lume finge, ora insieme gli mesce; e varia e vaga in cento modi occhi bramosi appaga.

$(G C \text { XIII 2O-2I })^{23}$

Indossata dalla Fortuna, la gonna cangiante - attributo non tradizionale - significava instabilità, incostanza. Ma non c'è dubbio che risultasse un po' inerte, ai fini dell'allegoria, l'insistenza sull'aspetto percettivo di questa mutevolezza, che invece l'attribuzione ad Armida valorizza pienamente. La gonna è infatti un perfetto equivalente figurativo dell' atteggiamento della maga seduttrice nell'accampamento cristiano: lo svariare da un colore all'altro ricorda, anche nel modulo sintattico (" or cerulea ed or vermiglia / diresti, ed or s'indora ed or verdeggia »; l'elenco è leggermente ampliato rispetto alla Liberata), l'illusionistica mutevolezza della maga, che produceva nei crociati uno sconcerto simile a quello che tocca a chi osserva il magico tessuto; mentre, allinterno della similitudine, la piccola correzione finale (" occhi bramosi") sostituisce " $i$ riguardanti ») non fa che rendere piu esplicita e moralmente 
connotata l'evocazione della folla che Armida aveva abbindolato con le sue «mille arti» ( $i$ «mille modis» ovidiani). D'altra parte, l'effetto del colore cangiante ("si ch'uom sempre diversa a sé la vede, / quantunque volte a riguardarla riede ») oggettiva quella mutevolezza di gusto in cui il Minturno individua una delle fonti del carattere camaleontico del bello (" ad un nomo istesso in diversi tempi sogliono piacere diverse cose $), \int$ so) e di cui dà prova nelle lodi di Laura quell'eminente Proteo che è il Petrarca. Bisogna inoltre osservare come la descrizione e la similitudine si ripartiscano le due facce complementari di questa ambiguità: da una parte la percezione (" diresti ... la vede ... a riguardarla riede »), dall' altra la rappresentazione ("si mostra ... si tinge ... finge ... mesce»); senza dimenticare che «fingere » $e$ "mescere» - oltre che termini di risonanza metapoetica peculiarmente tassiana - sono azioni tipiche della strategia di Armida, la seconda finalizzata proprio a ottenere un effetto cromatico di seducente indecifrabilità (GL IV 94 " $e$ 'l rossor de lo sdegno insieme n'esce / con la vergogna, e si confonde e mesce»). $\dot{E}$ chiaro, insomma, che questo attributo simbolico evoca non tanto un'instabilità sostanziale, oggettiva, quanto un fenomeno di ambiguità e di disturbo della comunicazione, pertinente come tale all' ambito della retorica e, in qualche misura, della poetica; e non ci sorprenderà constatare che a questo ambito rimandano anche $i$ cataloghi rinascimentali di iconologia, $i$ quali assegnano abiti cangianti - oltre che all'Inquietudine - alla Bugia, alla Simulazione e alla Loquacità. ${ }^{24}$ Qualche precisazione ulteriore viene dalla similitudine delle piume della colomba. Come segnalava già Guastavini, l'immagine deriva dal secondo libro del De rerum natura di Lucrezio, dove sta a illustrare la tesi democritea secondo cui il colore, qualità eminentemente mutevole ("Omnis enim color omnino mutatur in omnis », II 749), non è intrinseco alla materia, ma dipende dalla forma degli atomi e dall'incidenza della luce. ${ }^{25}$ Il contesto è dunque una teoria che potremmo chiamare relativista o prospettica ; e Tasso se ne ricorda bene quando riprende la similitudine, trasferendola dalla fisica alla retorica, nella sezione del dialogo Il Messaggero (stampato nel I582, rivisto nel I586) dedicata a comporre il ritratto del perfetto ambasciatore (\246):

Percioché, sì come le piume che sono nel collo de la colomba, benché sian sempre l'istesse e de l'istesso colore, ora paiono del colore de' rubini, ora s'assomigliano a' zaffiri, ora questi a gli altri colori sogliono mescolare secondo che variamente sono volti a la luce del sole, così l'azioni de gli uomini, tutto che siano l'istesse, posson prender diverse faccie, secondo che diversamente sono rappresentate a l'altrui considerazione : onde una azione istessa, diversamente posta al lume de la ragione, or buona pare, or rea, or mista, or degna di laude, or di scusa, or di vituperazione. ${ }^{26}$ 
La similitudine espone il fondamento retorico delle arti illusionistiche praticate da Armida : un medesimo oggetto può offrire ai suoi osservatori tante facce diverse perché le parole hanno il potere di modificare radicalmente l'aspetto delle cose, rappresentandole secondo prospettive moralmente opposte. Il regno in cui vige una tale libertà non è quello della verità ma quello dell'opinione, in cui può muoversi, con molta avvedutezza, solo chi, come l'oratore e l'ambasciatore, agisce per il bene della Città. E significativo che le teorie di Democrito, sfondo remoto di questa riflessione sul relativismo inerente alla retorica, siano evocate in modo esplicito, in una lettera del I588, a proposito della molteplicità che sarebbe tipica del verosimile in contrasto con l'unicità del vero : "le cose che hanno apparenza di vero (...) sono infinite, se non m'inganno, come gli atomi o l'imagini di Democrito. Ma la verità è una »; dove si dovrà vedere un'allusione, oltre che all' atomismo, alla teoria della conoscenza fondata sul concetto di eidolon 0 imago. ${ }^{27}$ Anche quello del verosimile è dunque un ambito caratterizzato da molteplicità, mobilità vorticosa (gli atomi) e, soprattutto, relativismo radicale; perché Tasso conosceva bene la descrizione delle idee democritee fornita da Aristotele nella Metafisica :

Inoltre essi dicono che molti degli altri esseri viventi delle medesime cose hanno impressioni sensoriali contrarie alle nostre e che addirittura ciascun individuo, considerato in rapporto con sé medesimo, della medesima cosa non sempre ha le stesse impressioni sensoriali. Non è dunque chiaro quali di queste siano vere e quali false : in realtà, le une non sono più vere delle altre, ma ambedue lo sono ad egual titolo. Per questo motivo, Democrito afferma che o non c'è nulla di vero o, almeno, che il vero ci rimane nascosto. ${ }^{28}$

Le percezioni dei fenomeni sono tante quante gli individui; ogni individuo ba nel tempo percezioni diverse dello stesso fenomeno : ritroviamo qui la radice filosofica di quella molteplicità prospettica che Tasso considerava tipica della bellezza e della poesia, o, meglio, della rappresentazione poetica della bellezza. Si deve dunque pensare che l'approfondirsi della dicotomia tra l'uno e il molteplice, tra stabilità e mobilità, metta in crisi anche il concetto di verosimile, uno dei cardini della poetica tassiana, separandolo irrimediabilmente dalla verità e riducendolo a una somma di opinioni mutevoli e contraddittorie? Per il momento, importa sottolineare che l'attribuzione ad Armida della gonna cangiante, accessorio la cui bellezza è una forma di illusione ottica, fa definitivamente di lei la signora di questo regno delle apparenze molteplici e della fluidità metamorfica; e che questo accade quando Tasso ha ormai esplicitato le ragioni filosofiche della sua diffidenza nei confronti di un ambito in cui l'attività del poeta non sembra distinguibile da quella del retore. Anche perché, va aggiunto, questo intervento non rimane isolato. La descrizione della gonna, 
prima di tutto, attiva lo stesso ordine di valori simbolici in due altre lussuose similitudini che accompagnano la donna fin dalla Liberata, quelle, improntate a svariante cromatismo, dell'arcobaleno e delle penne del pavone (GL $X V I_{24}$ e GC XIII 26). ${ }^{30}$ E probabilmente non è casuale che un fenomeno di cambiamento di colore sia attribuito nella Conquistata anche alle acque del mar Morto (".. tre volte a la celeste imago / il di si pinge e par cangiato in vista » GC XII 4) : una nota di illusionismo si aggiunge alle tradizionali connotazioni di sterilità infernale nella descrizione del luogo che è ora sede, oltre che di Armida, di una vasta comunità di maghi pagani. ${ }^{30}$ Infine, viene a inserirsi in questa isotopia anche una modifica del celebre ritratto morale di Armida, nel quale si insiste ora non tanto sul suo narcisismo, quanto sulla sua intrinseca mutevolezza, manifestata da uno sguardo che scoraggia ogni interpretazione definitiva : "....amò d'essere amata, odio gli amanti, / a cui fûr legge incerta i chiari lumi, / col variar de' suoi dolci costumi 》 (GC XIII 40). ${ }^{31}$

\section{Metamorfosi cortigiane}

Nel Messaggiero l'" artificio di far cangiar faccia a le cose 》 ( $\left.\int 247\right)$ non appariva moralmente connotato : stava all'oratore usarlo a buono o a cattivo fine, a beneficio o a danno del principe e dei suoi sudditi. Ma il Tasso degli ultimi anni sembra propenso a credere che di questa facoltà metamorfica si faccia soprattutto un uso perverso; e per illustrare gli esempi piu tipici di questa verità, quelli dell'adulatore e del cortigiano, recupera da una tradizione antica l'interpretazione moralistica del comportamento di due animali eminentemente proteiformi, il camaleonte e il polipo. Siamo rispettivamente nel dialogo Il Manso, overo de l'amicizia (I592), e nel Mondo creato (I593-1595), quindi nella fase estrema della produzione tassiana:

l'adulatore non è costante ne l'imitazione, ma mutabile in ciascuna forma e vario e sempre diverso da se stesso : co' cacciatori è cacciatore e giuocatore co' giuocatori, e musico fra' musici, lieto con lieti, mesto co' mesti, e in somma simile al camaleonte, il quale piglia tutti i colori de le cose che gli sono vicine.

$\left(\text { ll Manso } \int \mathrm{I} 9\right)^{32}$

Nè del polipo indietro i furti io lascio,

E i falsi inganni : che se mai s'appiglia,

A qualunque si sia marina pietra,

Egli repente si dipinge e veste

D’i' colori di quella, e lei rassembra. 
Armida e Proteo

Però se 'l pesce, che trascorre a nuoto, Da' sembianti ingannato in lui s'aviene, Pur duro sasso il crede in mare occulto, E di leggiero è sua rapina e cibo. Di tai costumi i lusinghieri accorti Son ne' palagi de' possenti Augusti O de' Regi sublimi, e 'n questa guisa S'inchinan pronti ad onorar l'altezza De la fortuna ; e trasmutar se stessi Sogliono in color mille, e 'n mille forme, Sì come l'uso, o 'l tempo, o come chiede La voglia del Signor e 'l suo diletto, Varïando tenor, sembianti e gesti, Parole e modi, e co' modesti insieme Sono modesti, e sospirosi in atto Co' più dolenti, e con gli allegri allegri, Protervi co' protervi. E legge e norma si fanno d'altrui senno e d'altrui gusto (...)

(Mondo creato $\mathrm{V} 405-27)^{33}$

La metafora del camaleonte proviene dal trattato di Plutarco sul vero e sul falso amico, dove si legge una lunga rassegna delle trasformazioni a cui si sottopone l'adulatore, per natura "molteplice e vario ", allo scopo di piacere a molte persone diverse; e Tasso aveva senza dubbio in mente anche l'opuscolo plutarcheo sulla pluralità di amici, in cui la stessa casistica sollecita invece l'evocazione di Proteo. ${ }^{34}$ La lettura morale del mimetismo del polipo è invece un luogo comune antico, che si fa risalire a Teognide e che ricorre anche nei luoghi citati di Plutarco; ma qui il Mondo creato segue alla lettera $i$ suoi modelli patristici, gli Esameroni di San Basilio e Sant'Ambrogio. ${ }^{35}$ Pur con qualche differenza - l'indole del camaleonte rivela debolezza e desiderio di piacere, quella del polipo è più insidiosamente strategica - le metamorfosi dell'adulatore e del cortigiano sono indizio della stessa malattia morale : una concezione della verità del tutto empirica e strumentale, calibrata sul «luogo» e sul " tempo », sulla molteplicità degli interlocutori e delle circostanze.

Non c'è dubbio che ad attirare l'attenzione di Tasso su questi topoi moralistici abbia contribuito non poco la sua esperienza infelice dentro e fuori dalla corte, la sensazione di incostanza che deriva tanto dal dovere di servire il proprio signore quanto dal bisogno di trovarne uno. Le lettere documentano con ampiezza l'insofferenza crescente del poeta per una condizione che impone di vivere "a gusto d'altri », " a le voglie ed a' commodi altrui», " a voglia d'altri »; e mostrano per converso l'importanza speciale che va acquistando ai suoi occhi, negli ultimi anni di peregrinazioni, la virtù stoica della costanza: 
" egli sa ... quanto mi dispiaccia ogni mutazione, ed ogni fama d'incostanza; ed, oltre a ciò, quanto m'incresca che mi sia data occasione d'esser discorde in alcuna cosa da me stesso", scrive nel I586 a Cristoforo Tasso; e nel I59I afferma a Fabio Gonzaga : "sono innamorato della costanza quanto alcun filosofo fosse già mai de la sapienza ». E a Marco Pio confessa nel Is 88 di recitare spesso "tra sé medesimo" (come, si direbbe, formula magica o preghiera filosofica) l'ode oraziana "Iustum et tenacem propositi virum»." D'altra parte, queste dichiarazioni si leggono in un epistolario che è nel suo insieme un esempio monumentale di retorica 'plurima', di espressione condizionata dall'occasione e dal destinatario. E in un momento isolato di buonumore e di verve metaforica Tasso arriva a descrivere la sua strategia epistolare nell'annoso «negozio "napoletano come una serie di metamorfosi : «Ma se Napoli è somigliata ad un grande e pigro cavallo ... foss'io come un vespone, affinch'ella si movesse prontamente a l'acume de le mie parole; o almeno fossi come una zanzara, che sonando la tromba, ed insieme pungendo, potessi risvegliarla». Movenza che riecheggia, come rivela il seguito della lettera ("Ma non vorrei tanto variare questi desiderii de la trasformazione, ch'io mi trasformassi in Anacreonte »), il celebre carme del corpus anacreontico in cui il poeta fantastica di trasformarsi nei vari oggetti che godono del contatto con la donna amata. ${ }^{37}$

Ma l'ideale etico della costanza è anche un ideale letterario. A questo proposito, nessun luogo è più esplicito della lettera a Scipione Gonzaga del ro gingno I590 : "Ho fatto, come sa, molte mutazioni ne' miei componimenti, e ne' costumi istessi; ma la costanza è cagione di tutta questa varietà.$^{3^{8}}$ Ripetendo il paradosso che esprimeva la moralità disillusa di Goffredo (" ché nel mondo mutabile e leggiero / costanza è spesso il varïar pensiero », GL V 3 e GC VI 3), ${ }^{39}$ Tasso descrive il vasto lavoro di revisione in cui si inserisce anche il rifacimento della Gerusalemme come una metamorfosi ispirata dalla costanza; formulazione che esprime da una parte la consapevolezza del carattere dinamico dell'opera letteraria, organismo sottomesso alle leggi del mondo e quindi suscettibile di continue modifiche; dall'altra l'ambizione pressante che l'opera trovi una sua definitiva stabilità, chiusura, indipendenza. Ma quello che più importa è che la condanna dell' incostanza cortigiana sembra portare con sé non solo il fastidio dichiarato per l'impegno estrinseco della poesia d'occasione, ma anche la messa in discussione dei presupposti teorici di un'attività letteraria che si è definita fin dai suoi inizi, e nel suo complesso, in rapporto all'orizzonte della corte. Il giovane Tasso mostra di avere piena consapevolezza del carattere convenzionale, mutevole e transitorio del mondo cortigiano, destinatario privilegiato del suo progetto epico : da cui quel compromesso tra regolarità e empirismo, tra culto della verità e strategia del consenso, "tra ricerca di statuti e coscienza della metamorfosi》, ${ }^{40}$ che si 
manifesta esemplarmente nei Discorsi dell'Arte Poetica. Con il passare del tempo questo equilibrio sembra rompersi, a favore di soluzioni ora appiattite sugli standard della retorica epidittica, ora irrigidite nell'autosufficienza della norma e dell' autorità. Ed è anche così che si spiegano il radicalismo e le contraddizioni del 'poema riformato': il progetto della Conquistata, che pure risulterà cosi affollata di omaggi cortigiani, nasce anche dal desiderio di affrancarsi dalla schiavitù dei desideri altrui, di erigere "con la prudenza e l'arte» un edificio intellettuale che sia sottratto ai condizionamenti del mondo. ${ }^{41}$ Questa ambizione contrasta con il nucleo più apertamente 'cortigiano' della poetica del giovane Tasso, quello dell" "uso»: «Ci ha alcune cose che 'n sua natura non sono né buone né ree, ma, dependendo dall'uso, buone e ree sono secondo che l'uso le determina"; consapevolezza relativistica (l'uso è "incerto e mutabile ») che tra l'altro aveva spinto l'autore della Liberata a bilanciare il rispetto della norma classica con una proficua frequentazione della letteratura contemporanea, quella romanzesca in particolare. ${ }^{42}$ Proprio in opposizione a questo eclettismo si elabora il programma di omerismo esclusivo della Conquistata, inteso a sottrarre il poema alla contingenza della sperimentazione letteraria per consegnarlo a una condizione di esemplarità estranea al tempo ("l'idea di poema»).

Ma si individuano in parallelo altre zone di crisi, strettamente connesse tra loro: la divaricazione tra il verosimile - l'ambito dell'opinione mutevole, della persuasione, delle convenzioni negoziabili socialmente - e il vero, inteso sempre più come conformità letterale a tradizioni autorevoli; l'opposizione tra una gestione del racconto prospettica, aperta a giudizi e adesioni emotive plurimi, e l'esigenza di istituire gerarchie morali non equivoche, che mette in questione anche la partecipe mobilità di un narratore dalle spiccate tendenze proteiformi; ${ }^{43}$ il contrasto tra l'aspirazione a raggiungere ogni lettore e il timore che la pluralità dei destinatari e dei gusti possa minare l'integrità, formale ed etica, del poema. Nel ritratto del polipo-cortigiano del Mondo creato abbiamo visto la descrizione delle arti di Armida convertirsi in una livida pagina di bestiario moralizzato; vediamo ora come la donna paghi la sua collusione con il mondo dell'artificio e dell'incostanza nel poema riformato, che sembra costruirsi in gran parte contro tutto ciò che essa rappresenta.

\section{Proteo legato}

Già nella Liberata il racconto della liberazione di Rinaldo (XIV-XVI) è ricalcato su quello della cattura di Proteo da parte di Aristeo che si legge nel IV libro delle Georgiche. ${ }^{44}$ Carlo e Ubaldo possono contare sull'ainto del 
Mago d'Ascalona come Aristeo su quello della madre Cirene; e tanto $i$ due crociati quanto il pastore visitano la fonte sotterranea di tutti i fiumi prima di ricevere dall'aiutante le istruzioni che, seguite scrupolosamente, li porteranno a concludere l'impresa con successo : tutto starà nel non lasciarsi ingannare dalle temibili «arti » che $i$ due avversari non dimenticano neppure nei momenti di più grave difficoltà (Georg. IV 440 "... ille suae contra non immemor artis »; GL XVI 43 «... costei, che ne la doglia amara / già tutte non oblia l'arti e le frodi »). Nella Conquistata il modello continua a essere attivo, ed è an₹i imitato più diffusamente nella descrizione degli antri subacquei, secondo una tendenza all' amplificatio che è tipica del poema riformato. ${ }^{45}$ Ma quello che più conta è che la traccia narrativa sia seguita fino in fondo, e che dopo la liberazione di Riccardo ad Armida tocchi di essere immobilizzata e incatenata come Proteo. Ecco le istruzioni del mago e il modo in cui i due crociati le mettono in pratica:

Ma s'ella, sue minacce aggiunte a' preghi, voi perseguisse, come suol, superba, non sia di voi chi per suo amor si pieghi, né per lusinga, o per querela acerba ; ma con più stretta mano allor si leghi per vostra mano, e non tra' fiori e l'erba. Voi da me di topazio infuso in Lete e d'adamante aspra catena avrete.

Ma de l'ascose insidie uscito Araldo, la cauta man gli avvolse entro a' capelli ; torcendo il viso al viso umido e caldo ed a' preghi, di fede ancor rubelli : e con quel laccio sì tenace e saldo legò le braccia e i piè fugaci e snelli co' nodi d'adamante e di topazio ; né fece altra di lei vendetta o strazio.

(GC XIII 70)

ma 'l laccio di topazi e d'adamanti non era sciolto, e quel che a' piedi il cinse disse : - Or securi andremo, e tu rimanti, perché senno e valor così t’avvinse : e vinta infernal fraude, onore avranno perfida lealtade e fido inganno - 
Nell'opera di Tasso non mancano le immagini di donne legate, e sono immagini di intensa sensualità : si pensi solo a Sofronia sul rogo, o a Silvia nelle mani del satiro. Ma qui tira tutta un'altra aria. Sulla scena sgombra si compie un rituale di composta e meticolosa brutalità : alla povera Armida non sono risparmiati né un trattamento dantescamente "aspro» (la perifrasi petrarchesca - Rvf. 53, I4 "La man' l'avess'io avolto entro' capegli»nobilita solo stilisticamente un gesto che mi sembra esemplato tanto sul rude assoggettamento della "donna pietra》 in Rime CIII 62-65, quanto sulla punizione del bestiale Bocca degli Abati in Inf. XXXII 103-5: "Io avea già i capelli in mano avvolti, / e tratti glien'avea più d'una ciocca, / latrando lui con gli occhi in giù raccolti»), né una descrizione di offensiva crudezza (" il viso umido e caldo »), che riduce alla sua essenzialità quasi ferina un dettaglio che era stato oggetto, in un precedente ritratto, di una memorabile promozione 'lirica' ("langue per vezzo, e'l suo infiammato viso / fan biancheggiando $i$ bei sudori più vivo», GL XVI I8). Ma si tratta, appunto, di un rituale, di una cerimonia allegorica : una soluzione formale di scontrosa arcaicità, del tutto estranea ai condizionamenti dell" "uso " contemporaneo, per la quale saprei indicare un precedente solo nell' Italia liberata di Trissino, già all'epoca il poema 'non letto' per antonomasia. ${ }^{46}$ Quanto al contenuto dell'allegoria, un compatto reticolo di allusioni dice chiaramente al lettore - perlomeno al lettore colto - che quella che si mette in scena qui è in primo luogo una replica del Trionfo della Pudicizia. Contrapposta con sarcasmo minaccioso ai soavi legami in uso nel regno d'Amore (Triumphus Cupidinis I 89-90 "Cesar, che 'n Egitto / Cleopatra legò tra' fiori e l'erba »), 47 i crociati si vedono infatti consegnare nientemeno che l'arma principe del trionfo di Laura:

D'un bel diaspro er' ivi una colonna,

a la qual d'una in mezzo Lethe infusa

catena di diamante e di topatio,

che s'usò fra le donne, oggi non s'usa,

legarlo vidi e farne quello stratio

che bastò bene a mille altre vendette

(Triumphus Pudicitie I 20-25)

Un prezioso concentrato di simbolismo lapidario, di cui Tasso conosce almeno la decifrazione proposta dal Castelvetro : "il diamante significa durezza contra Amore»; l'infusione in Lete vale "Dimenticanza, ché non è rimedio più potente contra Amore, che no 'I ricordare ", mentre la presenza del topazio si deve alla sua qualità di "refrigerativo del bollore del sangue, et per conseguente d'Amore ». ${ }^{48}$ A missione conclusa, poi, $i$ due crociati si congedano con un'altra citazione da Petrarca, ancora tra l'acre palinodia e il contrappasso: 
"perfida lealtate e fido inganno »è, in Triumphus Cupidinis IV I47, una delle definizioni ossimoriche di amore.

La vistosità delle allusioni non impedisce, tuttavia, che in questa scena Armida paghi, oltre a quelle di Cupido, le colpe di Proteo; che la sua cattura, in altre parole, si inserisca in modo del tutto coerente nel reticolo simbolico che abbiamo seguito fino a qui. In presenza di una segnaletica allegorica tanto esplicita può sembrare inutile ipotizzare palinsesti, moltiplicare $i$ significati. Ma è possibile dimostrare che il Tasso della Conquistata, che è tra l'altro un gran lettore di allegorie omeriche, non esita a riempire in verticale il suo testo, ad alimentarne lo spessore, convogliando materiali di diversa origine e valore attorno a nuclei simbolici elementari, quasi immagini ossessive : con un effetto non tanto di polisemia quanto di saturazione del senso. Uno di questi nuclei simbolici è sicuramente quello del 'legame', lo strumento che riduce il movimento a immobilità e la molteplicità a unità. La catena di diamante e di topazio si contrappone innanzitutto, come abbiamo visto, ai legami d'amore; e vendica, secondo la stessa logica, le vittime dell" "aurea catena di Armida, strumento insieme amoroso, magico e retorico ("Esce da vaghe labra aurea catena / che l'alme a suo voler prende ed affrena", GL IV 83 e GC V 82). ${ }^{49}$ Ma è interessante notare come gia in un sonetto dei primi anni ottanta alla dialettica del trionfo della pudicizia si sourapponga la simbologia di Proteo:

Più di saper che di contender vago,

Gualengo, io volgo or queste or quelle carte ove s'apprende il vero e 'nsieme l'arte che dal falso il distingue, e me n'appago; ma tu che fai ? Miri un cortese e vago ciglio, o la man ch'i cori incide e parte? o pur due trecce d'oro a l'aura sparte? Deh, non t'inganni Amor sofista e mago.

Ma da questi sì dotti antichi inchiostri d'ordire impara e tu sì forti nodi, che s'ei te prender vuol, tu lui n'avvolga; né rallentalo poi ben ch'ei si volga d'una in altra sembianza in vari modi, sin che nel proprio aspetto a te si mostri.

Amore assume la fisionomia del dio mutevole (Georg. IV 4II-I4: "Sed quanto ille magis formas se vertet in omnis, / tam tu, nate, magis contende tenacia vincla, / donec talis erit mutato corpore qualem / videris incepto 
Armida e Proteo

tegeret cum lumina somno ») ed eredita due sue qualifiche tradizionali, quelle di sofista e di mago. ${ }^{51}$ Ma questo significa che la logica moralistica del contrappasso resta in secondo piano rispetto a un'opposizione di diversa natura: quella, prettamente filosofica, tra due gradi diversi di verità. La capacità di distinguere il vero dal falso, frutto di un lungo tirocinio negli studi, si contrappone all'arte menzognera di Amore, che seduce le sue vittime creando una successione di mobili e vaghe apparenze; e non sarà difficile riconoscere nel "ciglio », nella "mano » e nelle "trecce d'oro » un compendio della fenomenologia petrarchesca del bello, intesa come molteplicità di frammenti sparsi e intercambiabili. Se bloccare le metamorfosi di questo Amore-Proteo significa, secondo tradizione, giungere alla verità, sarà allora perché la sapienza ereditata dagli antichi - una sapienza, si presume, filosofica e teologica - permette di arrestare il seducente flusso fenomenico del bello per isolarne l'essenza morale.

La posizione di questo Tasso studioso nei confronti dello svagato destinatario del sonetto anticipa perfettamente quella di Filagliteo, il sapiente che consegna la catena ai due crociati e li mette in guardia dalle insidie di Armida. Sarà dunque necessario tener conto delle qualità di questo personaggio per precisare ulteriormente il significato della cerimonia allegorica che egli organizza. Filagliteo è depositario di una immensa sapienza naturale, e nel libro precedente si esibisce in una rassegna enciclopedica dominata dall'assillo dell'unità : egli esplora il regno della natura "de l'Un ricercando alti vestigi 》 (GC XII 35). Se inoltre si considera che la metafora della catena indissolubile è usata insistentemente nei Dialoghi per indicare la coerenza e la coesione a cui devono aspirare le scienze della natura, risulterà chiaro che il destino della maga riattiva prima di tutto una valenza tradizionale del mito di Proteo, quella che faceva del dio legato l'immagine della verità naturale, la verità che risiede nei principi costanti della materia occultati dalla mobilità delle forme. ${ }^{52}$ Insieme alle metamorfosi della bellezza si blocca il fuire delle immagini naturali che erano il suo correlativo metaforico; la rappresentazione madrigalesca della natura, liquida, orizzontale, aperta, cede il posto all'esplorazione sotterranea e rigorosamente gerarchica dell" unica fonte 》 di tutte le cose.

Il nome parlante di Filagliteo deriva con ogni probabilità dal termine aristotelico philaléthes, che nel quarto libro dell' Etica Nicomachea (II27 b) designa chi pratica una virtù sociale equidistante dai viz̧i della dissimulazione e della millanteria. "' Ma il personaggio dà prova del suo 'amore della verità' soprattutto in ambito speculativo, e non solo nella filosofia naturale : lungo tutta la rassegna che occupa buona parte del libro XII egli è descritto come il garante di una percezione esatta, il nemico degli inganni, l'interprete razionale che sgombra la mente dalle ombre confuse dei sogni. Il suo rapporto con 
Armida ricalca dunque l'opposizione tra verità e menzogna sofistica che era abbozzata nel sonetto al Gualengo, e culmina nella medesima soluzione narrativa, per quanto attraverso un apparato allegorico più appariscente. Di questa simbologia è possibile precisare il valore e l'origine filosofica attraverso una citazione dal Porzio, il tardo dialogo (I593) dedicato al tema della virtù :

S. P. [Simone Porzio] : Le ragioni per opinione di Socrate legano ne l'animo l'opinioni in guisa che non possono fuggire, ma d'opinioni divengono scienze : e, se ciò è vero, l'intelletto di colui che sa è legato da le ragioni ; ma io avrei creduto più tosto che la nostra mente, quando ella è più adorna de l'abito de le scienze, sia più libera nel giudicare, e più vera estimo la sentenzia d'Aristotele nel settimo de la Filosofia de' costumi, che la mente sia legata da gli argomenti de' sofisti. D. C. [Dottor Calabrese] : 亡̀ come voi dite senza fallo; tutta volta la necessità, che portano seco le dimostrazioni di ciascuna scienza, sono così forti che potrebbero essere assomigliate a' nodi e a le catene del diamante : e gli antichi poeti, per quel Proteo che si transformava in tante sembianze, altro peraventura non vollero significare che 'l sofista trasmutabile in tutte guise, il quale al fine è legato da' lacci de la ragione. S.P. : Dunque la menzogna è legata da la verità, o 'l menzognero ; ma la verità dee rimaner disciolta e con le sue dimostrazioni adamantine legar più tosto gli altri che se medesima. (\$ 68-69) ${ }^{54}$

Nonostante la molteplicità di riferimenti, il modello preciso di questo passo è il Menone platonico, anch'esso dedicato al tema della virtù ; e naturalmente platonica è anche la dialettica tra ragione, opinione e scienza."

Questo sfondo filosofico permette di passare dalla decifrazione delle allegorie alla definizione del senso propriamente letterario dell'operazione tassiana. Notiamo, per cominciare, che nel Menone si trattava non di Proteo, ma delle mitiche statue di Dedalo : statue che prendono vita e che, se non sono legate saldamente, fuggono. Un altro mito incentrato su un movimento da bloccare, su una vitalità da disciplinare con un 'legame'; e non ci sorprenderà constatare, a questo punto, che anche esso torna più volte nell'opera tassiana, con una frequenza che contrasta con la sua diffusione relativamente modesta nella letteratura rinascimentale. ${ }^{56}$ Anche nella menzione dei "pié fugaci e snelli" di Armida c'è probabilmente il ricordo dell' agilità impertinente delle statue di Dedalo; e la sua riduzione all'immobilità è anche il gesto di un autore che si riappropria di una creatura troppo indipendente. Si può dire, infatti, che nella revisione dell'episodio Tasso mira soprattutto a definire rigorosamente il senso del suo testo, a predeterminarne la ricezione annullando ogni possibile ambiguità o pluralità di significato. Ė vero che, al contrario di Proteo, Armida è legata non per essere costretta a parlare, ma per essere ridotta al silenzio : e non solo nell'avvilente scena allegorica, ma per tutto il resto del poema, che 
rimane cosi privo del controcanto sentimentale che nella Liberata essa forniva all'irreprensibile carriera epica di Rinaldo. Ma cio non toglie che la sua cattura pretenda di essere una rivelazione della verità : una verità che ha $i$ colori netti e fermi dell'allegoria, non quelli cangianti della retorica.

Segnando il passaggio dal linguaggio dell' 'opinione' a quello della 'verità', il nuovo codice traduce nei termini di una scheletrica moralità la vicenda che il poema ha rappresentato fino a questo punto: Armida è un'incarnazione demoniaca di Amore " sofista e mago ", e nient'altro. È lo stesso procedimento che ̀̀ descritto nel Conte (』 I34) attraverso un altro impiego rivelatore, $e$ scopertamente metaletterario, della metafora del 'legare' : "Ora seguiamo a guisa di cacciatori le fiere in questa selva de l'invenzione, e prendiamo ciascuna al suo luogo e quasi ne la sua tana, e leghiamola con parole in modo ch'ella non si possa disciogliere »: ${ }^{57}$ "legare con parole 》 le fiere significa estrarre da ognuna di esse una qualità peculiare per trasformarla in « impresa ; " legare con parole» Armida significa ridurre la sua vitalità multiforme a una cifra simbolicamente compatta, a un emblema morale. Anche nella Conquistata, come nella Liberata, la donna ha il tempo di pronunciare la sua lunga e abilissima orazione suasoria, e di suggerire tanto a Riccardo quanto al lettore - non senza la sottolineatura partecipe degli interventi del poeta - l'esistenza di una prospettiva diversa da quella del dovere epico: ma la cerimonia allegorica che arriva subito dopo spegne l'eco del suo discorso con la perentorietà di una glossa autorevole, dell'unica esegesi autorizzata. Legare Proteo significa anche questo: opporre l'univocità impassibile dell'allegoria a quella retorica prospettica e a quella pluralità di voci che rendono cosi pateticamente conflittuale il mondo della Gerusalemme.

Matteo Residori 
I. L'uniforme cristiano e il multiforme pagano. Saggio sulla « Gerusalemme Liberata », Milano, I983.

2. Cfr. A. La Penna, Tasso e Ovidio. Aspetti della presenza di Ovidio nella " Gerusalemme Liberata », "Semicerchio », IX, I993, pp. 43-5 5 e S. Zatti, Il linguaggio della dissimulazione nella "Liberata », in L'ombra del Tasso. Epica e romanzo nel Cinquecento, Milano, I996, pp. I I I-45, in particolare le pp. I I 5-23. Sembra essere sfuggita ai commentatori quella che è forse la più vistosa tra le riprese dal poemetto ovidiano : cfr. GL V 70 : «Ella, che 'n essi mira aperto il core, / prende vedendo ciò novo argomento, / e su 'l lor fianco adopra il rio timore/ di gelosia per ferza e per tormento ; / sapendo ben ch'al fin s'invecchia Amore / senza quest'arti e divien pigro e lento, / quasi destrier che men veloce corra / se non ha chi lui segua e chi 'l precorra » e Ars amatoria III 593-96 : "postmodo riualem partitaque foedera lecti / sentiat : has artes tolle, senescet amor. / tum bene fortis equus reserato carcere currit / cum, quos praetereat quosque sequatur, habet ». Le citazioni sono tratte da Torquato TAsso, Gerusalemme Liberata, a c. di L. Caretti, Milano, i 979 e Ovidio, Amores, Medicamenta faciei femineae, Ars amatoria, Remedia amoris, a c. di E.J. Kenney, Oxford, I96I.

3. Zatti, Il linguaggio della dissimulazione cit., p. I I 9.

4. Le citazioni da Petrarca sono tratte dall'edizione a c. di M. Santagata, V. Pacca e L. Paolino, Milano, 1996.

5. Cfr. Zatti, Il linguaggio della dissimulazione cit., p. I 33 e n. Cfr. anche, a proposito dello spessore simbolico del personaggio nella Liberata, l'interessante studio di S. Volterrani, Tasso e il canto delle Sirene, "Studi Tassiani », XLV (1997), pp. 5 I-83.

6. È degno di nota che Briareo sia accostato a Proteo come simbolo di una stessa disposizione morale incostante e adulatoria nell'opuscolo di Plutarco Sulla pluralità di amici (Moralia, $95 \mathrm{E})$, che ci capiterà di citare anche in seguito.

7. Le sue parole riecheggiano quelle con cui la Fedra ovidiana dichiara a Ippolito la sua nuova passione per la caccia : Heroides IV 37 sgg. «Iam quoque, vix credes, ignotas mutor in artes ;/ (...) / In nemus ire libet pressisque in retia cervis / hortari celeris per iuga summa canes / aut tremulum excusso iaculum vibrare lacerto / (...) / Saepe iuvat versare leves in pulvere currus ... » (cito dall'edizione a c. di H. Bornecque, Paris, 1965); è interessante, ai fini del nostro discorso, che Tasso traduca ignotas ... artes con «forme insolite».

8. G. Gorni, Il "gran Sepolcro» di Gerusalemme. Sacro e finzione del sacro nel Tasso, «Compar(a)ison ", 2 (I 994), pp. 75-89, p. 78. Il libro citato di Rousset è naturalmente La littérature de l'âge baroque en France. Cyrce et le paon, Paris, 1954 (trad. it. Bologna, I985).

9. Gli studi citati sono, nell'ordine, A. Bartlett Giamatti, 'Proteus Unbound'. Some Versions of the Sea God in the Renaissance, in The Disciplines of Criticism. Essays in Literary Theory, Interpretation, and History, edited by P. Demetz, Th. Greene, L. Nelson Jr., New Haven, I968, pp. 437-75 ; D. Quint, Origin and Originality in Renaissance Literature. Versions of the Source, New Haven and London, 1983 ; M. Jeanneret, Perpetuum mobile. Métamorphoses des corps et des cuvres de Vinci à Montaigne, Paris, 1997.

ı. L'interpretazione di Proteo come sofista e mago è di origine platonica : cfr. Eutidemo $288 \mathrm{~B}-\mathrm{C}$; Ione 54I D ; è invece Luciano a interpretarlo come un ballerino di abilità prodigiosa, in un passo della Danza che godrà di grande fortuna presso i mitografi rinascimentali (cfr. Giamatti, 'Proteus Unbound' cit., pp. 462-63).

I I. Cfr. B.T. Sozzi, Per l'edizione critica dell' "Aminta», in Studi sul Tasso, Pisa, I954, pp. 44-45 n. e G. Da Pozzo, L'ambigua armonia. Studio sull" "Aminta» del Tasso, Firenze, 1983, pp. II9-20. 


\section{Armida e Proteo}

I 2. T. TAsso, Opere, a c. di B. Maier, Milano, i963, vol. I, pp. $205-6$.

I 3. Cito da T. Tasso, Rime, a c. di B. Basile, Roma, I994.

i 4. Cito da Virgilio, Opera, a c. di B. Mynors, Oxford, I969.

I 5. T. TAsso, Lettere, a c. di C. Guasti, Firenze, i $852-55$; lettera n. I423, del i 9 novembre I 592 (vol. V, p. I 24).

16. Poetica 1455 a. L'aggettivo che Tasso traduce con « di pieghevole ingegno » è eýplastoi : l'interpretazione del passo è dubbia anche per ragioni sintattiche, ma gli interpreti moderni sono in maggioranza concordi nel riferire l'aggettivo ai poeti e nell'attribuirgli il significato di «modellabili, adattabili, versatili» (cfr. la nota corrispondente nell'ed. a c. di C. Gallavotti, Milano, I974, pp. I62-63, che propone peraltro un'interpretazione diversa). Tra gli interpreti cinquecenteschi, Castelvetro volgarizzava con «trasmutabili» e parafrasava «cambievoli in ogni forma » : cfr. L. Castelvetro, Poetica d'Aristotele vulgarizzata, et sposta, Vienna, I 570 (rist. anast., München, I967), Terza parte, particella decima ottava, cc. 203 r e 208 r.

I 7. Lettere, n. I428, del I6 dicembre I 592 (vol. V, p. I 27).

I 8. T. TAsso, Dialoghi, a c. di E. Raimondi, Firenze, i958, vol. II, pp. 930-3 I.

I 9. «Bella non sete, o donna, / ma belli fate i cori, / belli gli animi vaghi e i vaghi amori ; / bello il soave sdegno, / belli i miei dubbi ancor, belli i tormenti, / le lacrime, i lamenti ; / bello il servir ne l'amoroso regno / con fortuna rubella, / per cui la morte e la vergogna è bella » (Rime 476).

20. Dialoghi, II, pp. 572 e 574 .

21. Lettere, n. 991 (vol. IV, pp. 76-77).

22. L'evoluzione del personaggio dalla prima alla seconda Gerusalemme ha già attirato l'attenzione degli studiosi; tra i contributi specifici ricordo L. Olini, Dalla «Gerusalemme Terrena » alla « Gerusalemme Celeste » : Rinaldo e Armida vs Armida e Riccardo, « Studi Tassiani », 33 (1985), pp. 69-87; C. Gigante, "Vincer pariemi più sé stessa antica». La Gerusalemme Conquistata nel mondo poetico di Torquato Tasso, Napoli, 1996 (in particolare le pp. 85-89) ; D. Della Terza, Armida dalla 'Liberata' alla 'Conquistata'. Genesi ed evolu₹ione del personaggio, nel volume miscellaneo Dal 'Rinaldo' alla 'Gerusalemme' : il testo, la favola, a c. di D. Della Terza, Sorrento, I 997, pp. 257-7 I ; G. Picco, "Idol si faccia un dolce sguardo e un riso »: Armida, in « Or s'indora ed or verdeggia ». Il ritratto femminile dalla 'Liberata' alla 'Conquistata', Firenze, I996, pp. $57-73$.

23. Cito dall'edizione a c. di L. Bonfigli, Bari 1934.

24. Cfr. le voci corrispondenti dell'Iconologia di Cesare Ripa, che ho consultato nell'edizione moderna a c. di P. Buscaroli, Milano, 1992.

25. De rerum natura II 799-805 : «Lumine quin ipso mutatur propterea quod / recta aut obliqua percussus luce refulget ; / pluma columbarum quo pacto in sole videtur, / quae sita cervices circum collumque coronat; / namque alias fit uti claro sit rubra pyropo, / interdum quodam sensu fit uti videatur / inter curalium viridis miscere zmaragdos » (cito dall'edizione a c. di C. Bailey, Oxford, I922). Negli ultimi anni della sua vita Tasso rilesse e postillò fittamente il poema lucreziano nell'edizione aldina del I5 I5, probabilmente in funzione della stesura del Mondo creato : nel volume, che ancora si conserva tra i postillati barberiniani, il verso II, 749 è sottolineato mentre l'intera descrizione delle piume della 
colomba è segnalata da un tratto di penna in margine. Cfr. B. Basile e C. Fanti, Postille inedite tassiane a un Lucrezio aldino, "Studi Tassiani », 25 (1975), pp. 75-168, in particolare p. I23; nello studio che precede l'edizione delle postille gli autori segnalano altri esempi dell'interesse tassiano per i meccanismi della visione e i fenomeni di illusione ottica (p. 98). I Luoghi osservati dal Mag. Giulio Guastavini, i quali il Tasso nella sua Gierusalemme ba presi, et imitati da poeti, et altri Scrittori antichi, apparsi per la prima volta nell'edizione genovese del Bartoli (1 590), si possono leggere anche nella ristampa anastatica del poema a c. di R. Peliti, Roma, I 966.

26. Dialoghi, vol. II, pp. 326-27. Una ripresa ellittica e puramente decorativa dell'immagine si ha invece ai vv. 5-6 del sonetto Qui giace Alfonso, e piantò il sacro alloro (Rime 623): "Veggonsi al sol ne l'una e gemme ed oro / splender qual varia suol piuma in colomba ».

27. Lettere, n. IoI I (vol. IV, p. 93) al cardinal Giovan Girolamo Albano; per la semantica specifica di imago si veda la voce del Thesaurus Linguae Latinae (Leipzig, 1934-1964), che cita numerosi esempi da Lucrezio.

28. Aristotele, Metafisica $1009^{\mathrm{b}}$ (trad. di G. Reale, Milano, I993); l'ultima frase è tradotta nel Malpiglio secondo ( $\$ I02) : «Democrito pronunziò che 'l vero fosse niente e oscuro" (Dialoghi, vol. II, p. 604). La conoscenza dell'opera aristotelica, che è tra l'altro per Tasso la principale fonte di informazioni sulle teorie dei presocratici, è attestata, oltre che da numerose citazioni, dalla postillatura, risalente con ogni probabilità agli anni giovanili, di un'edizione latina con il commento di Alessandro di Afrodisia : cfr. L. Capra, La 'Metafisica' di Aristotele nella lettura di Torquato Tasso, in Torquato Tasso e l'Università, a c. di W. Moretti e L. Pepe, Firenze, 1997.

29. Per le due similitudini Guastavini, seguito da tutti i commentatori, rimanda a Claudiano, De raptu Proserpinae, II 97-Io0, che peraltro ha con il luogo tassiano un'affinità solo tematica. Si può aggiungere che in Lucrezio la descrizione della coda iridescente del pavone segue immediatamente, e con la stessa funzione illustrativa, quella della colomba (II, 806-809), e che il Tasso postillatore accosta le due immagini in una nota marginale : «columbarum pluma et pavonis cauda» (B. Basile e C. Fanti, Postille inedite a un Lucrezio aldino cit., p. I 23).

30. La notizia è ricavata da Giuseppe Flavio, Guerra giudaica IV 84 : «Uno spettacolo meraviglioso è anche il mutamento del suo colore, che cambia tre volte al giorno col diverso riflettersi del colore del cielo » (cito dalla traduzione di G. Vitucci, Milano, 1974).

31. Ricordo il testo della Liberata (XVI 38) : « ... amò d'essere amata, odiò gli amanti ; / sé gradì sola, e fuor di sé in altrui / sol qualche effetto de’ begli occhi sui ». Alla luce di questi dati, mi sembra riduttiva l'interpretazione di Della Terza (Armida dalla 'Liberata' alla 'Conquistata' cit., p. 269) secondo il quale, con il trasferimento dalla Fortuna ad Armida, «la descrizione della gonna ... acquisisce una funzione estetica fine a se stessa ».

32. Dialoghi, vol. II, pp. $847-48$.

33. Cito dall'edizione a cura di G. Petrocchi, Firenze, 195 r.

34. Plutarco, Sul modo di distinguere l'adulatore dall'amico, VII-IX (Moralia $52 \mathrm{~A}-54 \mathrm{~A}$, in Oewvres morales, t. I, $2^{\mathrm{e}}$ partie, Paris, I 989 , pp. $9^{\mathrm{I}-96}$, in particolare p. 94) : «all'adulatore capita esattamente quello che capita al camaleonte, che adotta qualsiasi colore salvo il bianco »; il passo è tradotto, con esplicitazione del rimando a Plutarco, anche nel Conte, $\int 138:$ : Il camaleonte appresso Plutarco significa l’adulazione, perché prende i colori di tutte le cose vicine, se non il bianco, e perciò si dinota che la candidezza de' costumi non è imitata da gli adulatori» (Dialoghi, vol. II, p. I079); è interessante che Plutarco citi come esempio di adulazione ( $52 \mathrm{~B}-\mathrm{C}$ ) anche la dedizione alla caccia di Fedra innamorata, che è, 


\section{Armida e Proteo}

come abbiamo visto (cfr. la nota n. 7), modello della metamorfosi guerresca di Armida. Cfr. inoltre Plutarco, Sulla pluralità di amici, IX (Moralia 97 A, ibid., p. 228) : « Ci sarebbe bisogno di qualche Proteo ... che per magia fosse in grado di trasformarsi e di passare da una forma all'altra più volte in uno stesso istante, dedicandosi alla lettura con i dotti, coprendosi di polvere con i lottatori, inseguendo la preda con i cacciatori, ubriacandosi con i bevitori, facendo la campagna elettorale con gli uomi politici». Tasso lesse e postillò l'opuscolo sull'adulatore e l'amico nella versione latina di Erasmo, probabilmente durante la reclusione a Sant'Anna ; cfr. L. Chines, Tasso postillatore di Plutarco, in Torquato Tasso e l'Università cit..

35. Cfr. Basilio di Cesarea, Hex. ViI 3; Ambrogio, Hex. V 8 2i ; a questi luoghi, segnalati dal commento di Petrocchi, si può aggiungere un passo del De legendis libris gentilium dello stesso Basilio (IX) in cui l'animale è accostato al «sofista egiziano » Proteo come simbolo dell'instabilità che dipende dalla mancanza di saldi principi morali (cfr. l'ed. a c. di F. Boulenger, Paris, I 965, p. 59). Sulla fortuna classica del polipo come immagine di astuzia si veda il libro di M. Detienne e J.P. Vernant, Les ruses de l'intelligence. La mètis des Grecs, Paris, 1974, p. 45 sgg..

36. Le lettere citate sono rispettivamente ai nn. I065 (vol. IV, p. I42), I 348 (vol. V, p. 62), I 376 (vol. V, p. 87), 7 I I (vol. III, p. Iо2), I 303 (vol. V, p. 27) e 965 (vol. IV, p. 43) dell'edizione di Guasti. L'ode oraziana è la terza del terzo libro.

37. Lettere, n. I Ioo, a Ottavio Pisano, del I 589 (vol. IV, p. I7I). Cfr. Carmina anacreontea, a c. di M.L. West, Leipzig, I993, n. 22. L'attribuzione ad Anacreonte di questi testi di età alessandrina, che furono stampati per la prima volta e tradotti in latino da Henri Estienne nel I 554 , è normale fino all'Ottocento. Fanno parte del corpus anacreontico anche il carme che Tasso imita esplicitamente in Rime 1456 e quello citato nel Conte (\$209).

38. Lettere, n. I 25 I (vol. IV, p. 32 I).

39. La versione della Conquistata ( ... nel secol mutabile e leggero ») circoscrive più esplicitamente al mondo terreno questa condizione di mutevolezza.

40. Cf. G. Mazzacurati, Baldassar Castiglione e la prosopopea della corte, in Il rinascimento dei moderni. La crisi culturale del XVI secolo e la negazione delle origini, Bologna, 1985, pp. I49-207, p. 172. In questo importante saggio Mazzacurati individua una linea di forte continuità tra l'ideologia della corte e l'estetica elaborata dal giovane Tasso ; si veda anche Percorsi dell'ideologia cortigiana (ibid., pp. 209-35), dove si sottolinea tra l'altro l'importanza del ruolo di mediatore che ebbe in quest'ambito Sperone Speroni.

4I. Lettere, n. I64 (vol. II, p. I 29).

42. Discorsi dell'arte poetica, in Discorsi del'arte poetica e del poema eroico, a c. di L. Poma, Bari, I964, pp. 30-33.

43. Alludo agli studi fondamentali di R. M. Durling, The Figure of the Poet in Renaissance Epic, Cambridge (Mass.), I 965 e di E. Raimondi, Il 'poeta passionato', in Rinascimento inquieto (nuova edizione), Torino, 1994, pp. 33 I-48.

44. Per una storia della fortuna dell'episodio virgiliano di Aristeo e Orfeo nella letteratura rinascimentale cfr. D. Quint, Origin and Originality in Renaissance Literature cit., che contiene anche un'acuta analisi dell'episodio della Gerusalemme nelle sue due versioni (pp. 92-I32). Tasso sapeva naturalmente (lo attesta anche un passo dei Discorsi del poema eroico, in Discorsi ... cit., p. 73) che Virgilio imita a sua volta il IV libro dell'Odissea.

45. Cfr. GC XII I 3 : «Stupidi rimirâr gli umidi regni, / e tra spelunche chiuse acque stagnanti, / e sotto a' monti cavernosi e pregni, / senza luce o splendor, selve sonanti» e 


\section{Matteo Residori}

Georg. IV 363-65: «iamque domum mirans genetricis et umida regna / speluncisque lacus clausos lucosque sonantis / ibat». Per la tendenza all'amplificatio cfr. l'analisi stilistica di A. Di Benedetto, Dalla prima alla seconda "Gerusalemme», in Con e intorno a Torquato Tasso, Napoli, I996, pp. I 9I-24I, in particolare pp. 2 I 6 sgg..

46. Penso soprattutto all'episodio allegorico della liberazione di Areta nel V libro del poema, dove si rappresenta tra l'altro la cattura e l'incatenamento di due maghe dalle qualità propriamente proteiformi : "Quando si vider prese quelle maghe, / Mutorsi in acqua, per voler fuggire, / E quasi che li' uscir fuor de le braccia ; / Pur le ritenner fortemente ; e poi / Volsersi in foco, e in paventose serpi, / Volsersi in fumo, in nube, in tigre, e in orse, / Né mai però lascionle i buon guerrieri. / Ond'elle, visto che 'l cangiar figura / Non le giovava, ne la prima forma / Tornaro, e tutte liete si voltaro / A dolci prieghi, a parolette, e ciance ; / Ma parimente fur gettate al vento ; / Che la virtù del cielo havea sì chiuse / Le orecchie a quei Baron, che non sentiro / La forza, e 'l suon de i lor soavi accenti » (cito, modernizzando la punteggiatura, dalla c. $83 \mathrm{~V}$ del primo volume della princeps, stampata a Roma nel I 547 da Valerio e Luigi Dorici); scena che si può confrontare con quella già citata della tentata seduzione di Goffredo per constatare che neppure all'epoca della Liberata Tasso era insensibile al modello trissiniano, ma rifiutava la greve concretezza delle sue invenzioni a favore di una più sottile e coerente strategia metaforica. Il celebre giudizio tassiano su Trissino «mentovato da pochi, letto da pochissimi, prezzato quasi da nessuno »- detto in opposizione al successo del campione dell'« uso» moderno, Ariosto - si legge nei Discorsi dell'arte poetica (Discorsi... cit., pp. 22-23). Che l'operazione epica di Trissino sia condotta in aperta opposizione ai principi dell'ideologia e dell'estetica cortigiana è stato sottolineato opportunamente da A. Quondam, La poesia duplicata. Imitazione e scrittura nell'esperienza del Trissino, in Atti del Convegno di Studi su Giangiorgio Trissino, a c. di N. Pozza, Vicenza, I980.

47. Questi versi petrarcheschi sono citati nel Minturno $\left(\int_{42}\right)$ e nel Porzio $(\$$ I 88$)$ per esemplificare rispettivamente la potenza di Amore e gli effetti perniciosi dell'intemperanza (Dialoghi, II, pp. 928 e 1006 ).

48. Le Rime del Petrarca brevemente sposte per Lodovico Castelvetro, Basilea, ad istanza di Pietro de Sedabonis, I 582 , Parte terza, p. 257. Un esemplare di quest'opera conserva le tracce di una fitta postillatura tassiana, risalente con ogni probabilità all'arco degli anni I 585-I 594 ; sul margine della pagina citata Tasso trascrive « in Lete / infusa / catena di diamanti », e sotto, con riferimento all'interpretazione già citata di Castelvetro, «dimenticanza » : cfr. G. Baldassarri, Per un diagramma degli interessi culturali del Tasso. Le postille inedite al commento petrarchesco del Castelvetro, "Studi Tassiani », 25 (1975), pp. 5-74, in particolare p. 65. Le interpretazioni moderne di questa simbologia non si discostano molto da quella del Castelvetro : per un quadro esauriente si veda il commento di V. Pacca nell'edizione citata.

49. Si può ricordare che, negli stessi anni in cui Tasso lavora alla revisione della Gerusalemme, Giordano Bruno dedica a questo genere di ‘legami’ un opuscolo latino (il De vinculis) : sulle implicazioni letterarie della sua riflessione, che si avvicina per molti aspetti al trattamento tassiano del tema, cfr. L. Bolzoni, Le 'lien' magique de la beauté chez Giordano Bruno, «Carrefour», XVII (1996), pp. 9-25.

50. Il sonetto, che apparve a stampa già nel I 583 , è dedicato al gentiluomo Giulio Cesare Gualengo, con cui Tasso ebbe contatti soprattutto durante la reclusione a Sant'Anna.

5. Cfr. la nota n. 9.

52. Cfr. Malpiglio secondo \ I 8-19: «Laonde è un certo numero de le scienze che si posson legare con un legame, il quale è più saldo e di maggior prezzo che non son le catene di diamante »; "Il nodo de la necessità adamantino non può disciorsi »; "Troppo buon maestro sarebbe colui che m'insegnasse di far così preziosa catena » (Dialoghi, II, p. 575) e la 
lettera dedicatoria del Costante : «Le virtù ... sono collegate fra se medesime come le scienze, in guisa che non è alcun nodo più saldo o altra catena più forte, quantunque fosse di ferro o d'acciaio o d'altra più dura materia » (Dialoghi, II, p. 763). L'interpretazione tradizionale di Proteo come simbolo della varietà apparente del mondo naturale - per cui cfr. ad es. Eraclito, De allegoriis apud Homerum, 64-67 (ed. a c. di F. Buffière, Paris, 1989, pp. 69-72) e Plutarco, Moralia 97 B (ed. cit., t. I, ${ }^{\mathrm{e}}$ partie, p. 228 ) - sembra adombrata già in una lettera del I 579 (n. I 23 ; vol. II, p. 20) in cui Tasso scrive che « la materia prima ... è atta a ricevere la forma de la vite, de la palma, del leone, del destriero e de l'uomo o di che altro si sia».

53. La classificazione aristotelica è riecheggiata nel Porżio (\$ I57-158), dove la virtù in questione è chiamata « veracità » (Dialoghi, II, p. 996). Nonostante l'etimologia sia perspicua, la forma del nome - attestata, peraltro in una sola occorrenza (XII 39), fin dalla stampa pubblicata con l'approvazione tassiana (Roma, Facciotti, I 593) - non manca di suscitare qualche perplessità ; tanto più che nel Giudizio si trova la forma non palatalizzata «Filaliteo » (Del Giudizio sovra la sua Gerusalemme da lui medesimo riformata libri due, in T. TAsso, Prose diverse, a c. di C. Guasti, Firenze, I 875, pp. 479, 480 e 482 ; si deve comunque tener conto che il Giudizio attende una nuova edizione critica, anche in seguito al recente ritrovamento dell'autografo : cf. M.L. Doglio, Sull'autografo di Torquato Tasso «Del Giudicio sovra la sua Gerusalemme da lui medesimo riformata », «Lettere Italiane », XXXIII (198I), 3, pp. 389-99).

54. Dialoghi, II, pp. 965-966.

55. Menone 97 C-98 A : «Socrate - Come dici? Colui che sempre ha retta opinione, non dovrebbe riuscire sempre, fino a che almeno opini rettamente? Menone - Mi sembra necessario ; cosicché sono curioso di sapere, o Socrate, stando così la cosa, in che mai la scienza sia di maggior pregio della retta opinione, e perché siano diverse l'una dall'altra. S. - Sai perché ti fa meraviglia, o te lo dico io ? M. - Sì, dillo. S. - Perché non hai posto attenzione alle statue di Dedalo. Ma forse da voi non ce ne sono. M. - A qual fine dici questo ? S. - Perché anche queste, quando non siano legate, se la svignano e scappano ; se sono legate, invece, restano. M. - E allora ? S. - Possedere una delle sue opere sciolte non è cosa di grande valore, come uno schiavo che scappa - infatti non resterebbe ferma -; legata, è invece di molto pregio. Quelle opere sono infatti assai belle. Ma a qual fine dico queste cose? Riferendomi alle opinioni vere. Infatti, anche le opinioni vere, per tutto il tempo in cui rimangono, sono una bella cosa e producono ogni bene; ma troppo tempo non vogliono restare, e se ne fuggono dall'animo dell'uomo : sicché non sono di grande pregio, finché uno non le leghi, con la conoscenza della causa » (trad. di G. Reale, Milano, I99I). Il luogo di Aristotele a cui si allude nel Porzio è Etica Nicomachea I I45 a. Sia Della Terza (Armida dalla 'Liberata' alla 'Conquistata' cit., p. 27I) sia, in modo più articolato, Gigante ("Vincer pariemi più se stessa antica» cit., pp. 85-89) propongono di leggere la punizione di Armida come una replica di quella di Prometeo. La lettura che la associa a quella di Proteo mi sembra più coerente con i dati intertestuali e con gli altri elementi della caratterizzazione della maga ; tanto più che l'interpretazione del mito di Prometeo che si legge nello stesso Porzio $(\mathbb{\$} 7 \mathrm{I}-72)$ giunge alla conclusione che «Prometeo ancora come sofista è legato da Giove», cioè lo attrae nella sfera simbolica che è tradizionalmente associata a Proteo.

56. Particolarmente interessante è il passo del Minturno (\$ 20-22) in cui viene descritto come rovesciamento dell'arte di Dedalo l'artificio grazie a cui Ariosto ha dato una rappresentazione perfettamente 'statuaria' di Angelica e Olimpia legate allo scoglio - cioè l'antitesi della descrizione mobile e obliqua delle arti di Armida (Dialoghi, vol. II, pp. 9 2 I-22). Segue da vicino il modello platonico $L a$ Cavaletta ( 1585 ) $\$ Io : « Le mie (opinioni) non hanno tanta forza che possano legar gli uomini, quantunque di loro io vorrei fare quel che Dedalo già soleva de le sue statue, percioché elle, da le mie ragioni legate, di mobili divenissero stabili e ferme : quasi ardirei d'affermarvi ch'alcuna potesse tosto divenir scienza, se non temessi 


\section{Matteo Residori}

che questa paresse soverchia presunzione di me stesso (...) Quella che era dunque mia opinione disciolta ed errrante, ora spero di confermare con quelle ragioni che voi udirete » (Dialoghi, vol. II, p. 6r 8). Cfr. anche Rime I486, 4-6 e Ficino \50 (Dialoghi., vol. II, pp. 908).

57. Dialoghi, vol. II, pp. 1077-78. 